\title{
Chordoma of the Sacrum - A Case Report of Enbloc High Sacrectomy and Review of Literature
}

\author{
Suresh S Pillai ${ }^{a}$, Rameez Roshan K N $\mathbf{N}^{\mathrm{b}}$, Swaroop Sujath ${ }^{\mathrm{b}}$, Sunil Paul Pathrose \\ a. Senior Consultant spine surgeon, Baby Memorial Hospital, Calicut, Kerala, India; \\ b. Orthopedic Residents, Baby Memorial Hospital, Calicut, Kerala, India; \\ c. Consultant Spine surgeon, Spine centre, Keezhillam, Perumbavoor, Kerala, India
}

Published on $1^{\text {st }}$ June 2020

\begin{abstract}
"Coccydynia" unresponsive to a trial of conservative treatment should be evaluated promptly. Otherwise we may be fraught with the danger of serious complications. Sacral tumors are often quiescent to start with. They may achieve enormous size before they become symptomatic. Here is a case report and literature review of such a case in a 63 year old male, which was initially treated as coccydynia, till he became severely symptomatic. Chordomas are the most common primary tumor of the sacrum in adults. They are locally aggressive with immense potential for local recurrence and metastasis to lungs and other bones. If they get to the right hands at the right time, they are mostly curable.

Total sacrectomy (Enbloc resection) is the treatment of choice for sacral chordomas. In early stages, a low sacral resection all from behind is possible. Lesions extending higher than S3 requires high sacrectomy and lumbopelvic fixation depending on the integrity of the sacroiliac joints. High sacrectomy has high morbidity of permanent colostomy and bladder catheterization.
\end{abstract}

A 63 year old man presented with coccydynia of long duration and was diagnosed as chordoma of the sacrum upon imaging and image guided biopsy. He was treated with anterior, posterior and anterior surgeries in a single sitting, through a multi disciplinary approach.

Key Words: Chordoma, Enbloc Sacrectomy, Lumbo-pelvic Fixation, Physaliferous, Notochord Remnants

\section{INTRODUCTION}

$\mathrm{M}$ ost common tumors of the axial skeleton are metastases. Sacrum is no different. Primary tumors also arise in the sacrum from the bone, cartilage, nerve, marrow, or meninges. ${ }^{1}$ These lesions present with characteristic radiological and histological signatures, which aid in diagnosis and grading. Many systems like urogenital, anorectal, vascular structures, abdominal viscera, nerve roots, and plexuses converge at the sacrum. So lesions occurring at this diverse cross roads exhibit varying clinical presentations and treatment options. Most of the sacral lesions are quiescent to start with and remain so until they are gross enough to exhibit pressure effects on the surrounding structures. This would complicate the approaches to this tumors and the end results. Here we present such a case of sacral tumor, which was initially treated elsewhere as coccydynia until the patient was unable to sit or sleep due to severe pain. This case report highlights the importance of early imaging in back pain, which are recurrent or unresponsive to usual treatments, and the intricacies involved and disabilities associated with high sacral resections.

\section{CASE REPORT}

A 63-year-old male presented with low backache for 6-8 months' duration. He has been on treatment for coccydynia elsewhere with prescription for cushion while sitting along with medications without much relief. His pain aggravated recently for last 2 months. He had night pain which disrupted his sleep and he was unable to lie supine due to severe pain at the lower back. There was no relief of pain with NSAIDs and other pain medication. He had COPD, type 2 diabetes mellitus and he was a chronic smoker. He was evaluated further with MRI of the lower

Cite this article as: Pillai SS, N RRK, Sujath S, Pathrose SP. Chordoma of the Sacrum - A Case Report of Enbloc High Sacrectomy and Review of Literature. KJOrth. 2020 Jun 1;33(01):22-6.

$$
\text { Scan this QR Code }
$$

https://doi.org/10.52314/kjo.2020.v33i01.11 


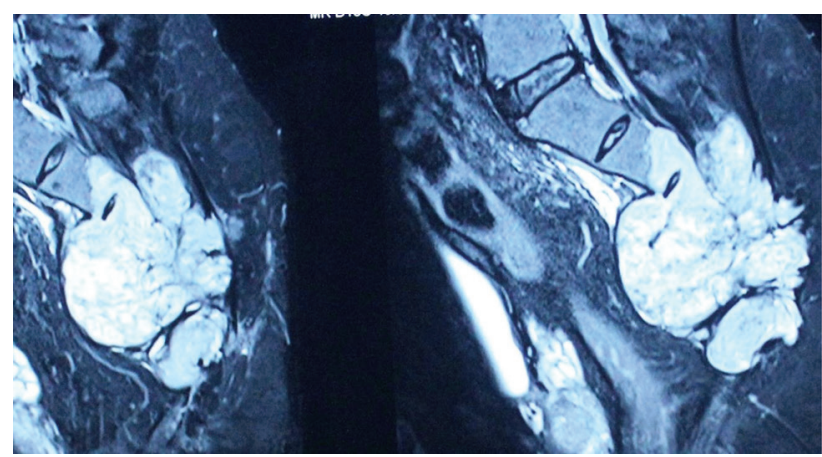

Figure 1. MRI showing the tumor extending to S2 body

back, which showed a tumor at the sacrum (Figures 1, 2, 3, 4). A CT-guided biopsy was done, which confirmed sacral chordoma. CT chest and abdomen did not show any evidence of metastasis. He underwent angioembolization of the tumor by interventional radiologist at out center. The tumor was extending up to S2 body and up to the sacroiliac joints. A total enbloc sacrectomy - high sacrectomy - was planned. The patient was counseled for the pros and cons of high sacrectomy especially about permanent colostomy and urinary catheter.

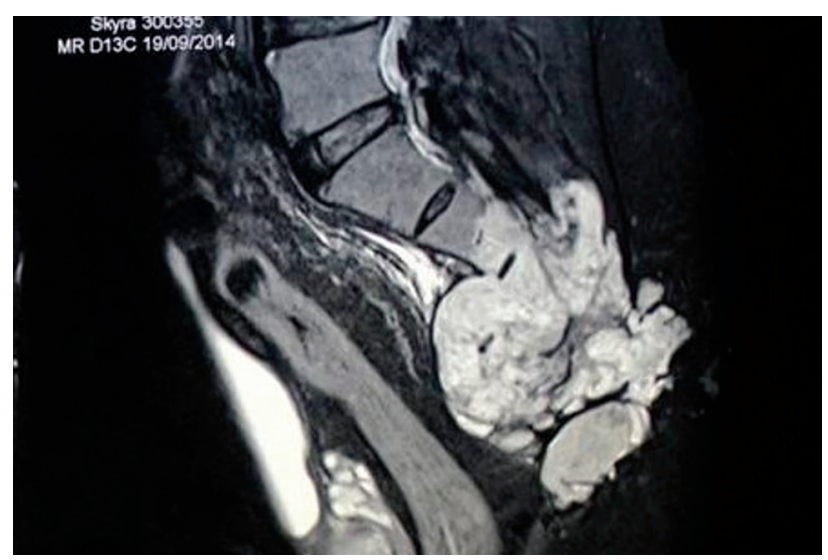

Figure 2. MRI Showing the extent of the tumor

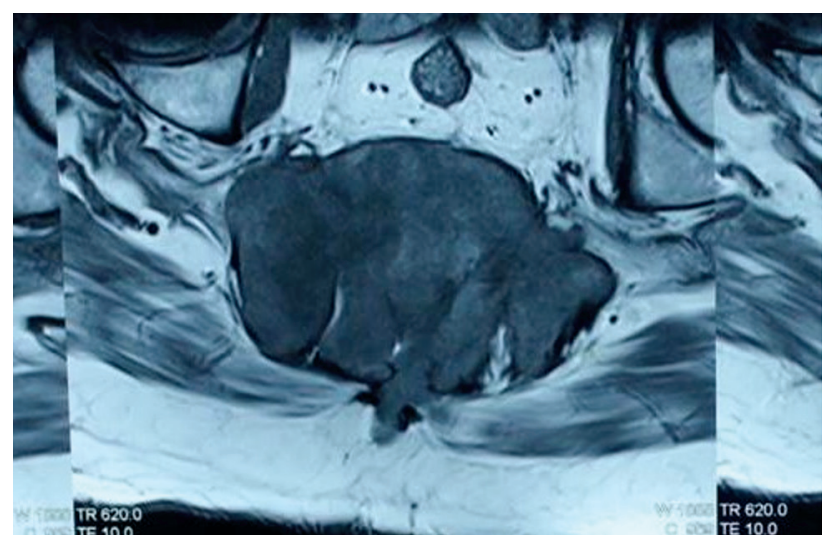

Figure 3. Axial MRI showing the extent of the tumor

For enbloc sacrectomy the patient was initially approached from behind in the prone position with isolation of the tumor and laminectomy of S1. The dural tube was isolated and resected at the lower border of S1 preserving

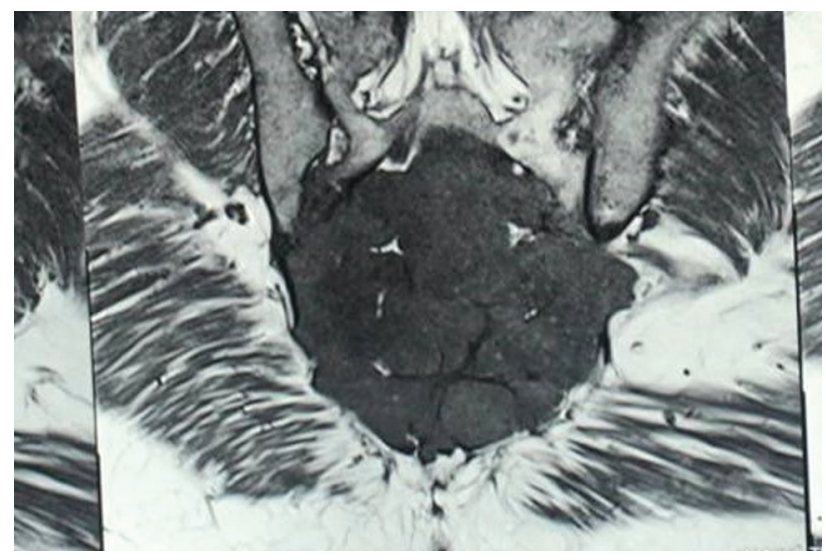

Figure 4. MRI showing the involvement of Sacro-iliac joint

both $\mathrm{S} 1$ roots. The dura was repaired with 6-0 Prolene. Lumbo-pelvic fixation was done with pedicle screws in L3, L4, \& L5 and into the iliac bones (Figures 5, 6, 7). The tumor was isolated till the coccyx. The wound was temporarily closed and the patient was made supine. The gastro surgeons did a midline laparotomy. Distal part of the rectum was resected, rest of the rectum was mobilized and kept in the abdomen for later permanent colostomy. Then with the help of plastic surgery the great vessels were mobilized. Internal iliac vessels were mobilized and retained and their branches were sacrificed. Ureters were also mobilized. Then, the patient was turned to lateral position. Osteotomy of the sacrum S1, S2 disc space was performed from behind protecting the vessels from the front. Sacrotuberous and sacrospinous ligaments were also sacrificed. Lower part of the right sacroiliac joint was removed and then the tumor was removed enbloc. A colostomy was performed by the gastro surgeon and a mesh was used to separate the pelvic cavity from the back. During the whole procedure he was transfused with fluid 6 units of PRBC and 4 units of fresh frozen plasma. Postoperatively, the patient was ventilated for five days. He was not weaning out on the ventilator and was restless when attempted to extubate. After extubation he was observed in the ICU for two days and then shifted to room. A clear fluid leakage was noticed from both anterior and posterior wounds once he was shifted to the

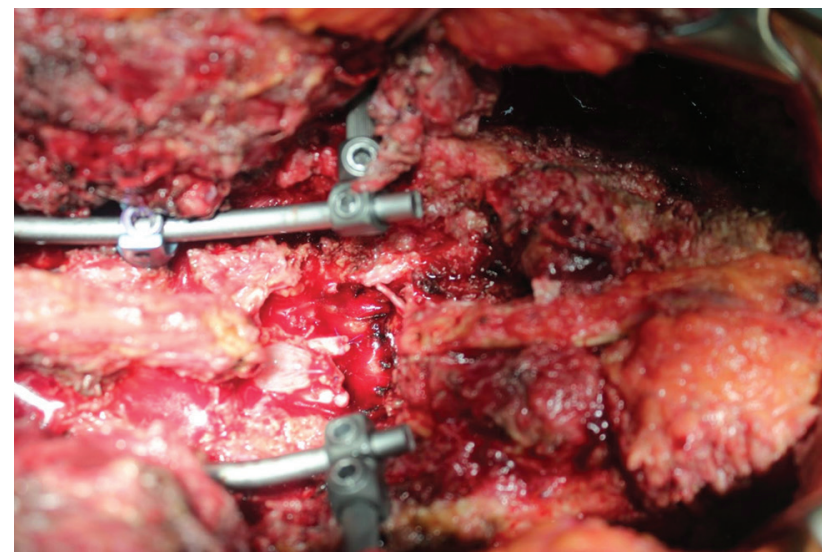

Figure 5. Intra operative picture showing lumbo-pelvic fixation insitu with isolation of the tumor from the posterior aspect 


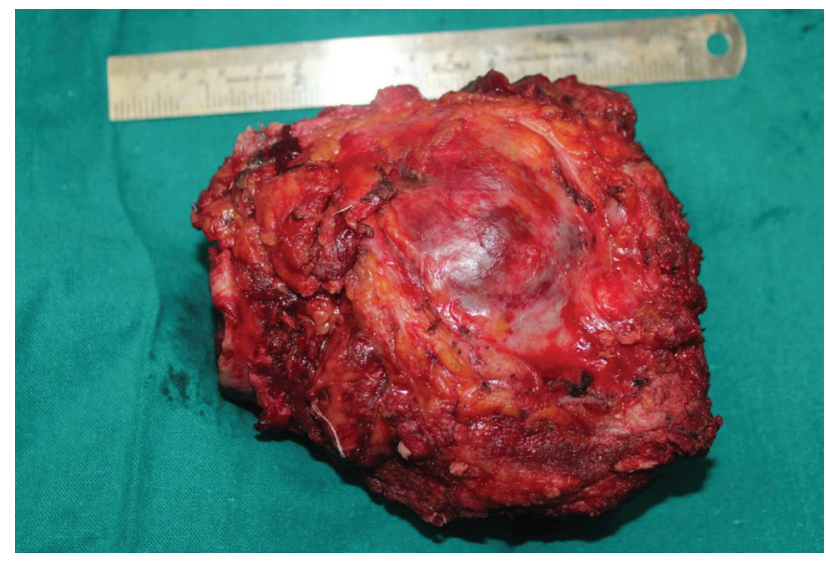

Figure 6. Removed tumor en mass

room. About 1.2 L of clear fluid was drained each day. Then, he developed abdominal distention, disorientation, and breathing difficulty. His PTI, INR, \& electrolytes were monitored. INR was only 70\%. A USG abdomen revealed cirrhosis of liver with splenomegaly and ascites (pre-operative LFT was normal). He was started on treatment for hepatic encephalopathy in consultation with Gastro Medicine Department. Fresh frozen plasma and albumin were transfused along with other medications. Then, he developed surgical site infection. Culture from the infected wound grew Acinetobacter (XDR). Also, he developed chest infection with Klebsiella (XDR) and the patient was put on culture-sensitive antibiotics. Even with these modalities, he was not improving. Fungal culture grew Candida and he was put on sensitive anti-fungal agent. By this time, the patient started improving, he started up sitting, moving his legs and eating.

Histopathological examination of the specimen revealed chondroid chordoma with margins free of tumor. By this time he was shifted back to the room again. With albumin \& FFP transfusions, his ascitic fluid leak was less than $10 \mathrm{ml}$ per day. Sutures removed from both wounds. The posterior wound started gaping, so the mesh was removed and the wound was packed with Betadine-soaked gauze for the granulation. VAC therapy was planned for further wound healing.

\section{DISCUSSION}

Most of the patients with sacral tumors present with severe local pain. Most of them are not able to sit for more than a few minutes, and sit on one buttock tipped to the side. Night pain, which wakes the patient up from his sleep is a common complaint. Weakness of leg, bowel and/or bladder dysfunction (39\%), sexual dysfunction (34\%). The average age of these patients are 55 years. These patients are evaluated radiologically and image-guided biopsies are preferred to avoid spillage of the tumor. Most often it is difficult to make out these lesions in plain radiography. CT scan/MRI are the main stay of evaluation. CT scan would provide osseous details and characteristic features of the tumor, ${ }^{2}$ while MRI provides detailed soft tissue

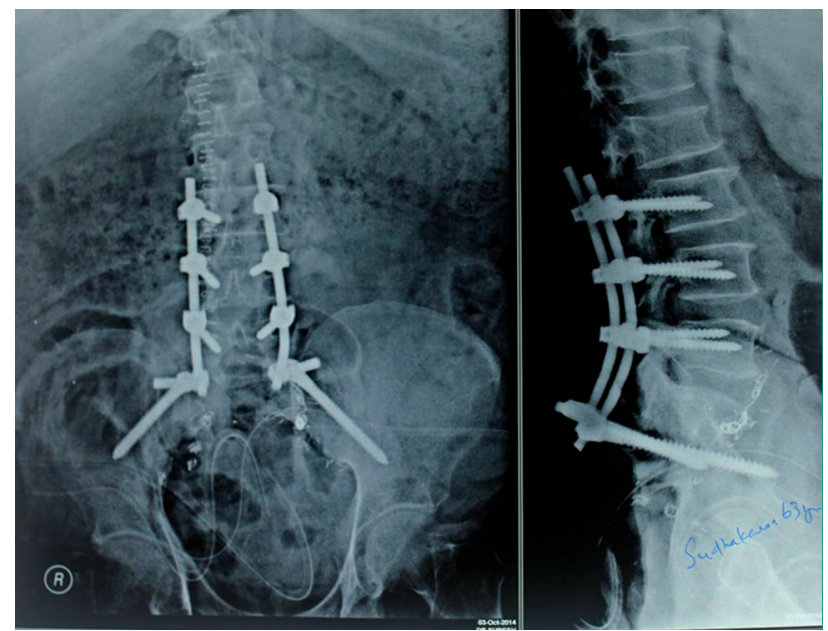

Figure 7. Radiograph showing lumbo-pelvic fixation

evaluation. CT scan would show cortical erosion, soft tissue extension, mineralization of the lesion all of which suggest malignant potential. CT scan also help in guided biopsies, which is essential for grading of the tumor. CT reconstruction helps in planning the surgery and $\mathrm{CT}$ of the chest can exclude possible metastasis. A whole body bone scan may be added to look for possible metastasis from these sacral tumors. MRI of the pelvis and sacrum would show extra-compartmental involvement of the tumor and aid in staging of the disease. ${ }^{3}$ These tumors moderately enhance with gadolinium. T1 weighted images show the extend of marrow replacement, which is an indication of its malignant potential. The composition and structure of the tumor are evident in T2, T1, \& contrast-enhanced pulse sequence comparisons. Bone scan has limited role in diagnosis of sacral tumors except for metastatic workup of these patients.

The most common primaries for sacral metastasis are lung, breast, kidney, prostate, GIT. There may be osteolytic or osteosclerotic lesions, or multifocal within the sacrum or in the adjacent part of the pelvis and spine. Chordomas are the most common primary sacral tumors in adults comprising approximately $40 \%$ of all sacral tumors and $50 \%$ of primary malignant osseous tumors of sacrum. ${ }^{1}$ These tumors arise from midline notochord reminiscence. ${ }^{4}$ More than half of the chordoma arise in the sacrum and about one-fourth occur in the cranio-cervical junction. They grow locally and invade the adjacent soft tissue. These tumors can metastasize to lung and other bones. They are prevalent more in the fifth and sixth decades of life with males affected twice as females.

Patients usually present with pain. A digital rectal examination might reveal the tumor in early stages before it grows huge in size to compress the surrounding structures and produce persistent symptoms. Histologically chordomas reveal pathognomonic physaliferous cells that are heavily vacuolated.

The main stay of treatment chordoma is enbloc resection of the tumor (without spillage) and reconstruction if 
necessary. Chemotherapy is ineffective. Proton beam radiation therapy may be tried along with surgery or alone in non-accessible areas. ${ }^{5}$ The recurrence rate of these tumors are surprisingly very high, up to $80 \%$ in some series and 5 year survival rate ranges from $45 \%$ to $70 \%{ }^{6}$ The recurrence and survival depends largely on surgical margins attained, the size of the tumor at presentation and the cephalad extend of the tumor., ${ }^{6,7}$ Surgical treatment provides significant pain relief to these patients and it is one of the primary goals of surgical treatment of chordomas.

Angioembolization is very useful when a surgical resection of sacral tumor is planned. This would help in providing better visualization, safer, meticulous surgery. Depending on the extend of the tumor sacral tumors repair partial or total sacrectomy. Tumors below S3 are resected all from behind and tumors above S3 has to be approached sequentially and with anterior and posterior approaches. ${ }^{6}$ Laparotomy is performed through anterior approach, the rectum is mobilized for later colostomy. The iliac vessels and ureters are mobilized and an omental flap along with a surgical mop is placed between the sacrum and the vessels to be retrieved from behind when the sacrum is resected afterwards. $^{8}$ In high sacral resection the dural tube is cut and ligated at S1 or S2 level with possible preservation of $\mathrm{S} 1$ and/or S2 nerve roots. Resection of the sacral nerve roots would cause bowel and bladder incontinence. So, a permanent colostomy and urinary catheter are imperative. A 2-cm margin of the tumor is preferable. The biopsy tract should be excised enbloc with the tumor whenever possible. The dural sac is ligated just caudal to the caudal most nerve root to be spared using 0 Silk and is over sewn with 4-0 Prolene. A Valsalva maneuver is performed to check the integrity of the dural closure. The sciatic notch and SI joints are exposed bilaterally. The sciatic nerve, adjacent pudendal nerve, and superior gluteal artery and vein are identified and protected. If sacroiliac joint is involved in the lesion, part of the ileum also should be included in the resection. The sacro-spinous and sacrotuberous ligaments are sectioned along with levator ani muscle. The plane between the sacrum and pre-sacral space is developed by blunt dissection beginning at the coccyx and proceeding up to the front of the sacrum. The sacrum is osteotomized at the planned cephalad level and laterally, approximately $2 \mathrm{~cm}$ from the tumor margin. The sacrum along with the tumor is then removed enmass. Rest of the pelvic contents are examined for residual lesions. Adequate blood and blood products should be arranged as this last stage of tumor resection can cause the greatest blood loss. If resection of the tumor included sacroiliac joint and compromise its function a reconstruction with spino-pelvic instrumentation should be performed with three levels of lumbar vertebrae and the ileum. The mop retained anterior to the sacrum should be removed and omentum retained to cover the potential space created by the sacrectomy. A Prolene mesh can be sutured to the pelvic bones to cover the pelvic floor. Gluteal flaps are rotated to enhance soft tissue coverage.

Urological, anorectal and sexual function may be compromised by sacrectomy. ${ }^{6}$ In general unilateral preservation of S2, S3, and S4 nerve roots or preservation of both S2 nerve roots and at least one S3 nerve root carries a high probability for retaining bowel, bladder, and sexual function. Exceptions to this rule are possible. Post sacrectomy ambulation depends upon the intactness of L5 on S1 nerve roots bilaterally and the integrity of sacroiliac joints. $50 \%$ of these patients require assistive device after high sacral resection. ${ }^{6} 40 \%$ of patients develop posterior wound complications.

\section{CONCLUSION}

Treatment of sacral tumors need multi-model and multidisciplinary approaches. Total sacrectomy is a challenging surgical endeavor. Resection via total or partial sacrectomy is the treatmental choice for most sacral tumors. Incisional biopsy or intra-lesional resection increases the risk of local recurrence. Transcutaneous CT-guided trocar biopsy is recommended. Proper patient selection is very important. Early diagnosis would limit the extent of sacral resection and the associated morbidities. So, early evaluation and proper imaging are recommended for patients presenting with coccydynia like symptoms.

\section{END NOTE}

\section{Author Information}

1. Dr Suresh S Pillai, MS (Orth) D. Orth, DNB (Orth), FIMSA, MNAMS, MCh (Orth), Fellow (Spine), FRCS (Glasgow)

Senior consultant spine surgeon, Baby Memorial Hospital, Calicut, Kerala, India

2. Dr Rameez Roshan K N, Orthopedic Resident (DNB) Orthopedic Residents, Baby Memorial Hospital, Calicut, Kerala, India

3. Dr Swaroop Sujath, Orthopedic Resident (DNB) Orthopedic Residents, Baby Memorial Hospital, Calicut, Kerala, India

4. Dr Sunil Paul Pathrose, MS Orth Consultant Spine surgeon, Spine centre, Keezhillam, Perumbavoor, Kerala, India

Conflict of Interest: None declared

\section{REFERENCES}

1. Gerber S, Ollivier L, Leclère J, Vanel D, Missenard G, Brisse H, et al. Imaging of sacral tumours. Skeletal Radiol. 2008 Apr;37(4):277-89.

2. Disler DG, Miklic D. Imaging findings in tumors of the sacrum. AJR Am J Roentgenol. 1999 Dec;173(6):1699-706. 
3. Enneking WF. A system of staging musculoskeletal neoplasms. Clin Orthop Relat Res. 1986 Mar;(204):9-24.

4. Dahlin BC, McCarty CS. Chordoma. Cancer 1952;5(6):1170-1178

5. Park L, Delaney TF, Liebsch NJ, Hornicek FJ, Goldberg S, Mankin $\mathrm{H}$, et al. Sacral chordomas: Impact of high-dose proton/photon-beam radiation therapy combined with or without surgery for primary versus recurrent tumor. Int J Radiat Oncol Biol Phys. 2006 Aug $1 ; 65(5): 1514-21$.
6. Hulen CA, Temple HT, Fox WP, Sama AA, Green BA, Eismont FJ. Oncologic and functional outcome following sacrectomy for sacral chordoma. J Bone Joint Surg Am. 2006 Jul;88(7):1532-9.

7. Fuchs B, Dickey ID, Yaszemski MJ, Inwards CY, Sim FH. Operative management of sacral chordoma. J Bone Joint Surg Am. 2005 Oct; 87(10):2211-6.

8. Diaz J, McDonald WS et al. Reconstruction after extirpation of sacral malignancies. Ann Plast Surg. 2003 Aug 1;51(2):126-9. 